\title{
Tuning the Re/Os Clock: Stellar-Neutron Cross Sections
}

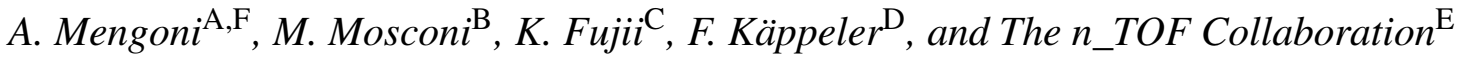 \\ A International Atomic Energy Agency, Vienna, Austria and CERN, Geneva, Switzerland \\ B Physikalisch-Technische Bundesanstalt (PTB), Braunschweig, Germany \\ ${ }^{C}$ INFN, Sezione di Trieste and Universitá degli Studi di Padova, Italy \\ D Forschungszentrum Karlsruhe, Germany \\ E CERN (www.cern.ch/ntof), Geneva, Switzerland \\ F Corresponding author. Email: a.mengoni@iaea.org
}

Received 2009 January 9, accepted 2009 March 12

\begin{abstract}
The neutron-capture cross sections of ${ }^{186,187}$ Os have been recently measured at the CERN neutron time-of-flight facility n_TOF for an improved evaluation of the Re/Os cosmo-chronometer. This experimental information was complemented by nuclear model calculations for obtaining the proper astrophysical reaction rates at $s$-process temperatures. The calculated results and their implications for the determination of the time-duration of nucleosynthesis during galactic chemical evolution is discussed.
\end{abstract}

Keywords: nuclear reactions, nucleosynthesis, abundances — galaxy: evolution — stars: neutron

\section{Introduction}

The use of the slow $\beta$-decay of ${ }^{187} \mathrm{Re}$ into ${ }^{187} \mathrm{Os}$ as cosmochronometer was proposed by Don Clayton in 1963 (see Figure 1). It was immediately noted that the neutron capture cross sections of the two $s$-only isotopes, ${ }^{186} \mathrm{Os}$ and ${ }^{187}$ Os would be key quantities for the determination of the $s$-process component of the ${ }^{187}$ Os abundance (Clayton 1964), and hence essential for isolating the radiogenic component. Estimates of these quantities, or of their ratio

$$
R_{\sigma} \equiv \frac{\langle\sigma(186)\rangle}{\langle\sigma(187)\rangle},
$$

were made from capture cross section systematics $\left(R_{\sigma} \approx 0.5\right)$, till the first direct measurements were performed in the 1980 (Browne \& Berman 1981; Winters \& Macklin 1982).

In parallel, it became clear that the experimental data had to be corrected for the basic formulation of the clock due to thermal effects in the stellar plasma. It turned out that the role of low-lying states in ${ }^{187}$ Os was particularly crucial for obtaining the $(n, \gamma)$ cross section at the temperatures of the $s$-process site, which are of the order of $3 \times 10^{8} \mathrm{~K}$. Under these conditions low-lying states are populated by excitation in the hot thermal photon bath. Therefore, the measured $(n, \gamma)$ results for the ground state have to be corrected for the contribution of the excited states to the effective stellar capture cross section.

This contribution complements improved experimental studies of the $(n, \gamma)$ cross sections of ${ }^{186,187}$ Os (Mosconi 2007; Mosconi et al. 2008; Segawa et al. 2008) and of the $\left(n, n^{\prime}\right)$ cross section of ${ }^{187}$ Os (Mosconi 2007) by an updated and consistent set of calculations to quantify the corrections for the effect of thermally populated nuclear states at the high temperatures of the stellar interior.

Direct information on capture rates from excited states is impossible to obtain by laboratory experiments. However, theoretical model calculations, which are necessary to evaluate the effective stellar cross sections of ${ }^{186}$ Os and ${ }^{187} \mathrm{Os}$, can be constrained by a number of experimental data. In this contribution the methods and informations used in these calculations are reviewed on the basis of recent experimental results, i.e. the measured $(n, \gamma)$ cross sections from the $n_{-}$TOF facility at CERN as well as the inelastic scattering cross section obtained at FZK Karlsruhe (Section 2.1). Up-to-date calculations with the Hauser-Feshbach statistical model are presented in Section 2.2 for different assumptions made in the evaluation of the model parameters. The implications of these results for the determination of the time-duration of nucleosynthesis (and ultimately of the age of the universe) are discussed in some detail in Section 3. It will become clear that the major uncertainty on the age determination based on this radioactive decay is now due to the modeling of the Galactical Chemical Evolution (Clayton 1988).

\section{Neutron Cross Sections}

\subsection{Measurements}

The neutron-capture cross sections of ${ }^{186,187}$ Os have been recently measured at the CERN neutron time-of-flight facility $\mathrm{n}_{-}$TOF. Details of the measurement technique and data analysis have been reported elsewhere (Mosconi 2007; Mosconi et al. 2008). Here it suffices to report the final Maxwellian-averaged capture cross sections, MACS, for the two isotopes in Table 1. 


\begin{tabular}{|c|c|c|}
\hline \multirow[b]{2}{*}{ 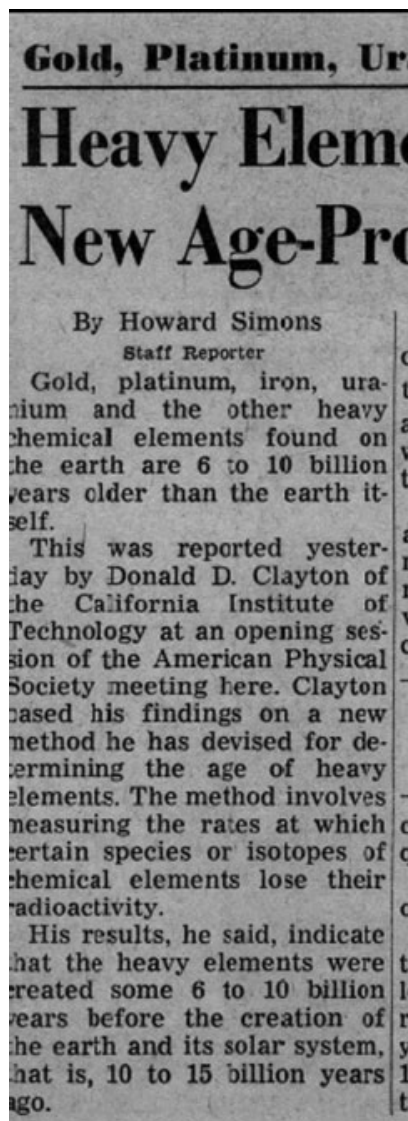 } & in I & \multirow[b]{2}{*}{ 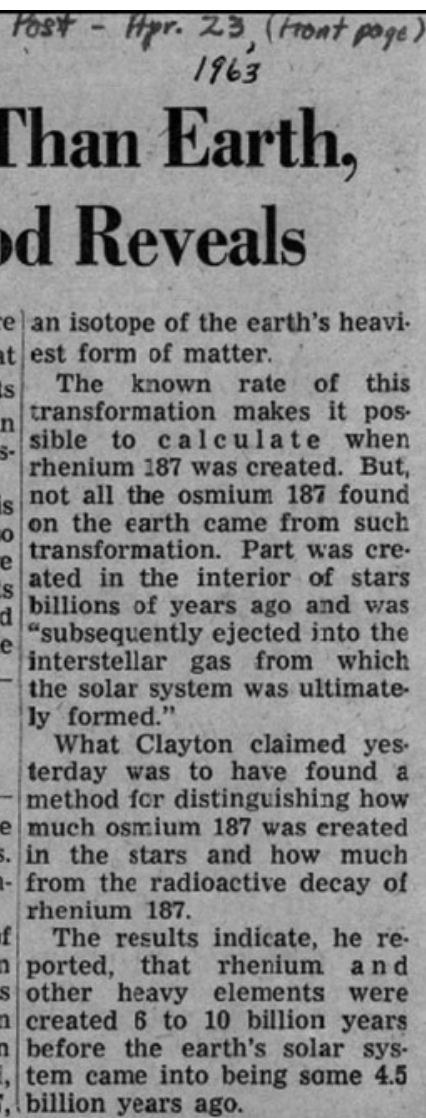 } \\
\hline & $\begin{array}{l}\text { OntS } \\
\text { If Ciayton's findings are } \\
\text { correct it would mean that } \\
\text { the earth's heavy elements } \\
\text { are as ald as the galaxy in } \\
\text { which the earth's solar sys- } \\
\text { tem is lacated. } \\
\text { Clayton told reporters his } \\
\text { age-determining method is so } \\
\text { new that other scientists have } \\
\text { not had time to challenge its } \\
\text { validity. Though he expressed } \\
\text { complete confidence in it, he } \\
\text { Scientists suspect 'hit. } \\
\text { and-run' viruses moy be } \\
\text { cause of cancer. Page A3. } \\
\text { conceded that "there will be } \\
\text { questions" about his findings. } \\
\text { Essentially, Clayton's meth- } \\
\text { od works this way: } \\
\text { One particular isotope of } \\
\text { the heavy element rhenium } \\
\text { loses about 10 per cent of its } \\
\text { radioactivity every } 6 \text { billion } \\
\text { years. In doing so, rhenium } \\
\text { 187, as the isotope is called, } \\
\text { transforms into osmium 187, }\end{array}$ & \\
\hline
\end{tabular}

Figure 1 The front page of the Washington Post of 23 April 1963 where the proposal of using the Re/Os clock by Don Clayton was reported after the announcement at the meeting of the American Physical Society. The story, while written in non-specialistic language, correctly reports the basic principle on which the clock is based (courtesy of Don Clayton, 2008).

Table 1. Maxwellian-averaged capture cross sections at $k T=30 \mathrm{keV}$ and cross-section ratios, $R_{\sigma}$

\begin{tabular}{lccc}
\hline & $\mathrm{A}$ & $\mathrm{B}$ & $\mathrm{C}$ \\
\hline${ }^{186} \mathrm{Os}$ & $414 \pm 17$ & $418 \pm 16$ & $438 \pm 30$ \\
${ }^{187} \mathrm{Os}$ & $969 \pm 32$ & $874 \pm 28$ & $919 \pm 43$ \\
$R_{\sigma}$ & $0.43 \pm 0.02$ & $0.48 \pm 0.02$ & $0.48 \pm 0.04$ \\
\hline
\end{tabular}

A, Mosconi et al. (2008); B, Winters \& Macklin (1982); C, Browne \& Berman (1981).

The energy dependence of the cross section is important in this context since the parametrization of the model calculation, which is necessary to deduce the stellar rates, can be validated by comparison of the calculations with the measured cross sections. The relevant energy range for this comparison from $0.1 \mathrm{keV} \leq E_{\mathrm{n}} \leq 1 \mathrm{MeV}$ is perfectly covered by the high-resolution results of the n_TOF measurement. The new n_TOF data are shown in Figure 2 together with the results of previous capture cross section measurements and the updated calculations.

\subsection{Calculation}

In the mass range $A \approx 185$, the Hauser-Feshbach statistical model (HFSM) is best suited in the energy range of interest for this work. The model relies on the assumption that the reaction proceeds through formation of a compound nucleus and its subsequent statistical decay, following well established statistical properties. In particular, the nearest level spacings correspond to a Wigner distribution and the width for neutron decay to a PorterThomas distribution. The decay probabilities for $\gamma$-ray emission can be deduced from time-reversal invariance (detailed balance) and the Brink-Axel hypothesis.

Then, the simple HFSM formula holds:

$$
\sigma_{\mathrm{n}, \gamma}\left(E_{\mathrm{n}}\right)=\frac{\pi}{k_{\mathrm{n}}^{2}} \sum_{J, \pi} g \frac{\sum_{l s} T_{\mathrm{n}, l s} T_{\gamma, J}}{\sum_{l s} T_{\mathrm{n}, l s}+\sum_{l s} T_{\mathrm{n}^{\prime}, l s}+T_{\gamma, J}} W_{\gamma, J}
$$

where $E_{\mathrm{n}}$ is the incident neutron energy, $k_{\mathrm{n}}$ the wave number for the neutron-nucleus relative motion, $s=\frac{1}{2}$ the intrinsic spin of the neutron, and $l$ the orbital angular momentum of the relative motion. The statistical weighting factor for target nuclei in states of spin $I$ and compound states of total angular momentum $J$ compatible with spin and parity conservation laws is indicated by $g_{J}=(2 J+1)(2 s+1)^{-1}(2 I+1)^{-1}$. Finally, $W_{\gamma}$ is a factor which takes the different statistical fluctuation properties of the $\gamma$-decay channel compared to the neutron elastic (n) and inelastic $\left(\mathrm{n}^{\prime}\right)$ channels into account. 

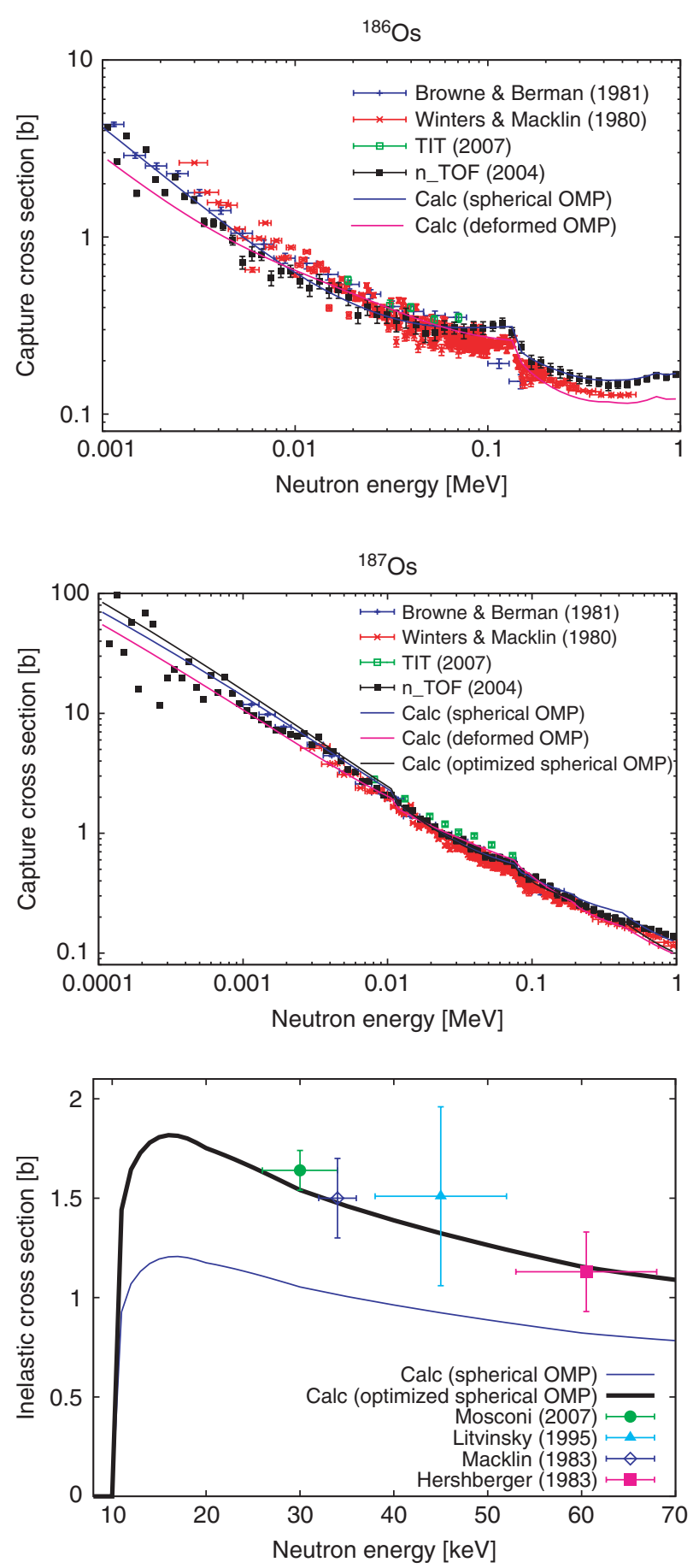

Figure 2 Top and Middle: neutron-capture cross sections of ${ }^{186} \mathrm{Os}$ and ${ }^{187} \mathrm{Os}$ as a function of neutron energy. Calculations were performed in this work within the HFSM theory, as described in the text. In the legend TIT refers to Segawa et al. (2008) and n_TOF to Mosconi et al. (2008). Bottom: the inelastic cross section for the first excited state in ${ }^{187} \mathrm{Os}$ at $9.75 \mathrm{keV}$ (Mosconi 2007).

The nuclear input data for the HFSM theory can be calculated using different nuclear structure and deexcitation models. It is here that the various HFSM approaches used so far differ among each other. Examples of widely used approaches to the HFSM parameterization for nuclear astrophysical applications are those of Holmes et al. (1976), Harris (1981) and the most recent versions of NON-SMOKER (Rauscher \& Thielemann 2001), MOST (Arnould \& Goriely 2006), and TALYs (Koning, Hilaire \& Duijvestijn 2005). Most of these references include also HFSM computer codes for calculation of reaction cross sections. A repository of parameters and systematics of nuclear structure quantities can be found in the 'RIPL' initiative $^{1}$.

The present calculations of the neutron capture cross sections of ${ }^{186}$ Os and ${ }^{187}$ Os were performed using the standard optical model parameters (OMP) of Moldauer (1975) and of the code TALYS. The level density parametrizaion is that of Gilbert and Cameron with parameters, which are adjusted to reproduce the low-lying cumulative number of levels available from the data library ENSDF $^{2}$ at low excitation energies and the average level spacings at the neutron binding energy deduced from the n_TOF cross section measurements. The parameters for the $\gamma$-ray transmission coefficients were adopted from photo-neutron cross section data for the giant dipole resonance and the methodology used in TALYS.

Additional calculations were performed using a deformed optical potential, considering the coupling of the rotational states in the target such as the $0^{+}, 2^{+}, 4^{+}$states in ${ }^{186} \mathrm{Os}$ and the $1 / 2^{-}, 3 / 2^{-}$, and $5 / 2^{-}$states in ${ }^{187} \mathrm{Os}$. The coupling strengths were deduced from the groundstate quadrupole and octupole deformations, which are also available from the ENSDF data library.

Both sets of calculations are included in Figure 2. The calculated cross sections were normalized to reproduce the MACS at $k T=30 \mathrm{keV}$ given in Table 1 . The plots of Figure 2 show that the use of the deformed OMP does not result in a better reproduction of the capture cross sections in the energy range of interest, but that the cross sections obtained with the spherical OMP are closer to the experimental data. This can be due to an overestimation of the coupling strength deduced from the static quadrupole deformations. In the actual situation, a smaller coupling strength could be more appropriate for the description of the experiemental data, a situation comparable to that described by a spherical OMP set.

A similar behaviour is also seen in the comparison with the experimental data for the inelastic cross section. Again, the deformed OMP calculations do no seem to improve the results obtained with spherical OMPs. In fact, a small adjustment of the spherical OMPs seems produce the best results, for the neutron capture as well as for the inelastic channel. This adjustement consists in a simple reduction of the strength for the real part of the spherical potential from $V_{0}=46 \mathrm{MeV}$ to $V_{0}=43 \mathrm{MeV}$.

As a conservative estimate of the uncertainties related to the OMP, the deviation of the cross sections calculated with the three OMP sets from the mean value was adopted in the evaluation of the stellar enhancement factors.

\footnotetext{
${ }^{1}$ RIPL-2 2007, see http : / / www-nds. iaea . org/RIPL-2.

${ }^{2}$ ENSDF, Evaluated Nuclear Structure Data File 2008, see http: //www-nds. iaea. org/nsdd.
} 


\subsubsection{Stellar Enhancement Factors}

The reaction rates under stellar conditions have to be evaluated by due consideration of the possibility that lowlying excited states can be thermally populated in the involved nuclei. Neutron capture rates are especially sensitive to this effect because the cross sections for excited states may well differ from that of the ground state.

The capture cross section of excited states can be modeled as for the ground state. There are, however, additional inelastic scattering channels, which have to be considered. In particular, the so-called super-elastic channel where the incident neutron is inelastically scattered to a lower state in the target, may contribute significantly. In the above HFSM equation, the transmission coefficients for the inelastic channels, $T_{\mathrm{n}^{\prime}, l s}$, must also include the open super-elastic channels. Comparison of the model calculation with experimental data for the inelastic scattering cross section provides a good benchmark for the neutronnucleus interaction used to determine the transmission functions.

As a consequence of the compound nucleus reaction mechanism the compound nuclear states formed by capture on excited states are the same (except for total angular

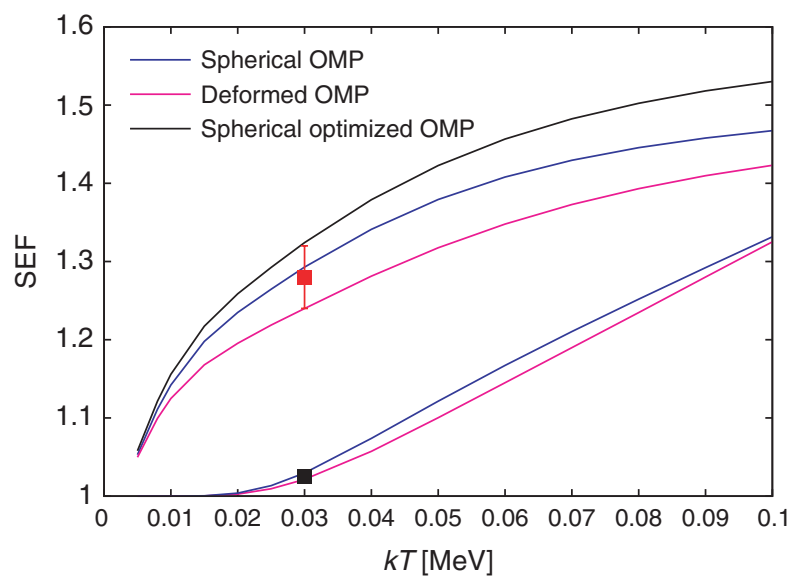

Figure 3 Stellar enhancement factor (SEF) for the ${ }^{187} \mathrm{Os}(n, \gamma)^{188} \mathrm{Os}$ reaction. Three types of calculations are shown, using different sets of OMP, standard values from Moldauer (1975), a deformed optical model with channel coupling, and a spherical OMP with optimized parameters. The average value at $k T=30 \mathrm{keV}$ has been adopted and the deviations from the mean was assumed to represent the associated uncertainties. momentum) as those populated by capture on the groundstate. Since the modeling of the reaction cross section for the ground-state can be tested against experimental capture cross sections measured in the laboratory, the constraints from this comparison can help reduce, at least in principle, the uncertainties related to the evaluation of stellar cross sections. In practice, the thermal effects are expressed by the so-called stellar enhancement factor (SEF), defined as $\langle\sigma\rangle^{*}=S E F \cdot\langle\sigma\rangle^{\text {lab }}$, where $*$ and 'lab' indicate the MACS (i.e. averaged over a population of excited states) and the laboratory (ground-state) cross sections, respectively.

The SEFs of ${ }^{186}$ Os and ${ }^{187}$ Os represent crucial corrections with respect to the analysis of the Re/Os cosmochronometer. Especially the stellar cross section of ${ }^{187} \mathrm{Os}$ is strongly influenced by the presence of low-lying excited states (Woosley \& Fowler 1979). Figure 3 shows examples of SEF calculations for this isotope.

\section{Tuning the Clock}

In order to establish a chronology based the cosmoradiogenic production of ${ }^{187} \mathrm{Os}$, one needs to develop a proper GCE model describing the production rate of ${ }^{187} \mathrm{Re}$. This program was started by Clayton (1988). However, the implications of the different uncertainties of the $\mathrm{Re} / \mathrm{Os}$ cosmo-chronometer can be analyzed with respect to their impact on the galactic age (or on the time-duration of nucleosynthesis) using the schematic model for an exponentially decreasing production of ${ }^{187} \mathrm{Re}$ with time proposed by Fowler \& Hoyle (1960). The discussion of the various effects is summarized in Table 2 , which refers to the uncertainties of the galactic age due to the Re/Os abundance ratios and the nuclear quantities entering the model.

The uncertainty of the SEF-30 value of ${ }^{187}$ Os was estimated to $\pm 4 \%$ from the difference of the results obtained with a spherical and a deformed optical model potential for the neutron-nucleus interaction. This corresponds to a $17 \%$ uncertainty for the contribution of the excited states to the overall cross section. While this is justified for the well studied case of ${ }^{187}$ Os, in general SEF corrections can be estimated with uncertainties of $\approx 5 \%$ if the capture cross sections for the ground-state (lab) are known with sufficient accuracy and if the other model parameters in HFSM calculations can be fixed by measured quantities.

Table 2. Uncertainties of the Re/Os cosmo-chronometer associated with the Re/Os abundance ratios and the nuclear-physics input

\begin{tabular}{|c|c|c|c|}
\hline Quantity & Value & Uncertainty (Gyr) & Reference \\
\hline${ }^{186} \mathrm{Os} /{ }^{187} \mathrm{Re}$ abundance ratio & $0.2845 \pm 0.0071$ & & Faestermann (1998) \\
\hline${ }^{187} \mathrm{Os} /{ }^{187} \mathrm{Re}$ abundance ratio & $0.2254 \pm 0.0057$ & & Faestermann (1998) \\
\hline Total effect of abundances & & 0.49 & \\
\hline$t_{1 / 2}{ }^{187} \mathrm{Re}$ & $41.2 \pm 1.12$ & 0.29 & Galeazzi et al. (2001) \\
\hline MACS ratio $R_{\sigma}(\mathrm{lab})$ & $0.427 \pm 0.023$ & 0.40 & Mosconi et al. (2008) \\
\hline SEF-30 ( $\left.{ }^{186} \mathrm{Os}\right)$ & $1.025 \pm 0.005$ & 0.04 & \\
\hline SEF-30 ( $\left.{ }^{187} \mathrm{Os}\right)$ & $1.28 \pm 0.04$ & 0.24 & \\
\hline Total combined uncertainty & & 0.74 & \\
\hline
\end{tabular}


It is interesting to note in closing that the various components of the uncertainty on the age add up to less than 1 Gyr. In other words, it appears that the uncertainties on the nuclear physics quantities as well as on the observed abundances, are contributing a relatively minor component to the uncertainty of the age determination based on the Re/Os clock. It is, however, all the most clear that at this point a proper GCE modeling of the production rate of ${ }^{187} \mathrm{Re}$, and/or of its destruction by astration (Arnould, Takahashi \& Yokoi 1984, and references therein), need to be accounted for in order to determine a meaningful chronology based on this interesting decay. A work which has been initiated (Clayton 1988) and that can be now exploited and brought to a much improved degree of accuracy.

\section{Acknowledgments}

It is an honour and a pleasure to dedicate this contribution to Roberto Gallino. Some of us have been collaborating with Roberto over the course of a few decades and a small contribution on 'age' might perhaps be appropriate as a small present at the occasion of his 70th birthday. Buon Compleanno Roberto!

\section{References}

Arnould, M. \& Goriely, S., 2006, NuPhA, 777, 157

Arnould, M., Takahashi, K. \& Yokoi, K., 1984, A\&A, 137, 51

Browne, J. C. \& Berman, B. L., 1981, PhRvC, 23, 1434

Clayton, D. D., 1964, ApJ, 139, 637

Clayton, D. D., 1988, MNRAS, 234, 1

Faestermann, T., 1998, Proceedings of the 9th Workshop on Nuclear Astrophysics, Ringberg Castle, Tegernsee (Garching: Max Planck Institute for Astrophysics), 171

Fowler, W. A. \& Hoyle, F., 1960, AnPhy, 10, 280

Galeazzi, M. et al., 2001, PhRvC, 63, 014302

Harris, M., 1981, Ap\&SS, 77, 357

Holmes, J. A., Woosley, S. E., Fowler, W. A. \& Zimmerman, B. A., 1976, ADNDT, 18, 305

Koning, A., Hilaire, S. \& Duijvestijn, M. C., 2005, AIPC, 769, 1154 Moldauer, P. A., 1975, PhRvC, 11, 426

Mosconi, M., 2007, PhD Thesis, University of Karlsruhe Mosconi, M. et al. (The n_TOF Collaboration), 2008, JPhG, 35, 014015

Rauscher, T. \& Thielemann, F.-K., 2001, ADNDT, 79, 47

Segawa, M. et al., 2007, PhRvC, 76, 022802

Winters, R. R. \& Macklin, R. L., 1982, PhRvC, 25, 208

Woosley, S. E. \& Fowler, W. A., 1979, ApJ, 233, 411 